\title{
Long-Term Results of Treatment for Critical Limb Ischemia
}

\author{
Takuya Miyahara, MD, PhD, ${ }^{1}$ Masamitsu Suhara, MD, ${ }^{1}$ Yoko Nemoto, MD, ${ }^{1}$ Takuro Shirasu, MD, ${ }^{1}$ \\ Makoto Haga, MD, ${ }^{1}$ Yasuaki Mochizuki, MD, ${ }^{1}$ Mitsuru Matsukura, MD, ${ }^{1}$ Takafumi Akai, MD, PhD, ${ }^{1}$ \\ Ryosuke Taniguchi, MD, PhD, ${ }^{1}$ Masaru Nemoto, MD, PhD, ${ }^{1}$ Satoshi Yamamoto, MD, PhD, ${ }^{1}$ \\ Ayako Nishiyama, MD, PhD, ${ }^{1}$ Akihiro Hosaka, MD, PhD, ${ }^{1}$ Katsuyuki Hoshina, MD, PhD, ${ }^{1}$ \\ Hiroyuki Okamoto, MD, PhD, ${ }^{1}$ Kunihiro Shigematsu, MD, PhD, ${ }^{1}$ Tetsuro Miyata, MD, PhD, ${ }^{2}$ \\ and Toshiaki Watanabe, MD, PhD ${ }^{3}$
}

From 2001 to 2012, arterial reconstruction was performed in 306 out of 497 limbs (62\%) with critical limb ischemia. The reasons for non-vascularization include high operative risk (36\%), extended necrosis or infection (20\%), and technical issues (15\%). Cumulative patency and limb salvage in collagen disease were significantly worse compared to arteriosclerosis obliterans. Cumulative limb salvage, amputation free survival (AFS), and major adverse limb event and perioperative death (MALE + POD) in patients with end-stage renal disease (ESRD) were significantly worse compared to patients without ESRD, but not significant with regards to graft patency. Our finding suggests that aggressive arterial reconstruction provides satisfactory long-term results in critical limb ischemia so long as case selection for revascularization is properly made. (This article is a translation of J Jpn Coll Angiol 2014; 54: 5-11.)

Keywords: arteriosclerosis obliterans, peripheral arterial disease, critical limb ischemia, arterial reconstruction

\section{Introduction}

The only method that markedly reduces symptoms of critical limb ischemia (CLI), such as rest pain and ulcers/

${ }^{1}$ Division of Vascular Surgery, Department of Surgery, The

University of Tokyo, Tokyo, Japan

${ }^{2}$ Vascular Center, Sanno Medical Center, International University of Health and Welfare, Tokyo, Japan

${ }^{3}$ Surgical Oncology, Department of Surgery, The University

of Tokyo, Tokyo, Japan

Received: July 21, 2015; Accepted: July 22, 2015

Corresponding author: Takuya Miyahara, MD, PhD. Division of Vascular Surgery, Department of Surgery, The University of

Tokyo, 7-3-1 Hongo Bunkyo-ku, Tokyo 113-8655, Japan

Tel: $+81-3-5800-8653$, Fax: $+81-3-3811-6822$

E-mail: tmiyahar-tky@umin.ac.jp

This article is a translation of J Jpn Coll Angiol 2014; 54: 5-11. This report was presented at Panel Discussion 1: 'Therapeutic strategies for CLI and their limits' of the 54th Annual Meeting of Japanese College of Angiology (October 2013, Tokyo). necrosis, is revascularization. It, including bypass, has been routinely performed. However, recently, a nitinol stent for the superficial femoral artery became commercially available, and endovascular therapy (EVT) for CLI has been indicated for an increasing number of patients. On the other hand, the widespread use of EVT in the femoral and crural artery regions may lead to occlusion of the collateral pathway through post-EVT stent occlusion, and such lesions may extend to the peripheral area. Actually, patients with the rapid progression of ischemia after EVT have been referred to the Department of Vascular Surgery.

The incidence of arteriosclerosis obliterans (ASO), which comprises the greater portion of patients with peripheral artery disease (PAD), has recently increased. However, this is a part of systemic arteriosclerosis. In particular, recently, the number of high-risk patients, including diabetics and those on maintenance dialysis, has increased. For treatment, it is important to examine treatment invasiveness in accordance with the prognosis or grade of risk and separately select bypass from EVT or combine the two procedures. We have positively performed bypass for CLI to achieve limb salvage. In particular, the introduction of a non-dissection method ${ }^{1)}$ has improved the accuracy and safety of vascular anastomosis below the popliteal artery. In some patients, it was impossible to indicate revascularization, and, finally, amputation was selected. In others, limb salvage could not be achieved due to protracted/extended infection despite revascularization. In this study, we investigated treatment strategies for CLI and limitations by clarifying whether or not revascularization for CLI is appropriate and reviewing the results of treatment.

\section{Subjects and Methods}

The subjects were 408 patients (497 limbs) admitted to our department between January 2000 and December 2012 and diagnosed with grade III or IV CLI according to 
Table 1 Characteristics of patients

\begin{tabular}{lccc}
\hline & $\begin{array}{c}\text { Vascularization } \\
(260 \text { cases, 306 limbs) }\end{array}$ & $\begin{array}{c}\text { Non-vascularization } \\
(170 \text { cases, 191 limbs) }\end{array}$ & P value \\
\hline Ages (years) & 68.8 & 68.9 & 0.95 \\
Male:Female & $183: 77$ & $117: 53$ & 0.73 \\
Etiology (collagen disease) & $8 \%$ & $14 \%$ & 0.07 \\
Fontaine IV & $82 \%$ & $87 \%$ & 0.11 \\
Rutherford 6 & $15 \%$ & $26 \%$ & $<0.01$ \\
Smoking & $60 \%$ & $56 \%$ & 0.41 \\
Hypertension & $81 \%$ & $75 \%$ & 0.16 \\
Dyslipidemia & $30 \%$ & $26 \%$ & 0.26 \\
Diabetes mellitus & $65 \%$ & $66 \%$ & 0.68 \\
IHD & $56 \%$ & $62 \%$ & 0.11 \\
CVD & $27 \%$ & $26 \%$ & 0.82 \\
ESRD & $40 \%$ & $42 \%$ & 0.58 \\
RD & $44 \%$ & $53 \%$ & 0.07 \\
Malignancy & $16 \%$ & $22 \%$ & 0.09 \\
\hline IHD: ischemic heart disease; CVD: cerebrovascular disease; ESRD: end-stage renal disease; RD: respiratory & &
\end{tabular}

the Fontaine classification. They consisted of 285 males and 123 females, with a mean age of 68.8 years ( 22 to 99 years). According to the Fontaine classification, the grade was evaluated as III in 82 limbs and IV in 415. The subjects were divided into two groups: a group in which revascularization was performed, and a group in which it was not conducted. We examined whether or not revascularization is possible, compared the patient background and prognosis, and reviewed the results of surgery in the revascularization group and long-term results.

To evaluate whether or not revascularization is possible, angiography was performed before surgery, excluding patients with an unfavorable general condition, including renal dysfunction, and those requiring emergency amputation due to infection or necrotic lesions involving an extensive area. Patients with acute arterial occlusion related to the exacerbation of ASO were excluded. As a rule, as grafts for revascularization, prosthetic grafts were used when the peripheral anastomotic site was located above the knee, and autologous veins were used when it was located below the knee. Furthermore, EVT was indicated for iliac/femoral artery lesions, as a rule. Concomitant diseases before surgery were diagnosed according to the criteria prepared by Rutherford et al. ${ }^{2)}$ Treatment results within 30 days after surgery were regarded as early results, and the subsequent results as long-term results. The results were compared between the revascularization and non-revascularization groups using Student's t-test and the chi-square test. Concerning long-term results, the cumulative rate was calculated using the Kaplan-Meier method, and compared between the two groups using the log-rank test. Furthermore, freedom from the major adverse limb event + perioperative death (MALE + POD) and amputation-free survival (AFS) were established as endpoints. Cox's proportional hazard analysis of risk factors influencing them was conducted.

\section{Results}

Of the 408 patients with CLI (497 limbs), revascularization was performed in 260 (306 limbs, 62\%), but not in 170 (191 limbs, 38\%). The patient background is shown in Table 1. In the revascularization group, the mean age was 68.8 years, and the male-to-female ratio was 183:77. According to the Rutherford classification, 45 limbs (15\%) were classified as Category 6. Primary diseases consisted of ASO in 265 limbs, Buerger's disease (thromboangiitis obliterans, TAO) in 15, and angiitis (collagen disease) in 26. In the non-revascularization group, the mean age was 68.9 years, and the male-to-female ratio was 117:53. According to the Rutherford classification, 49 limbs $(26 \%)$ were classified as Category 6. Primary diseases consisted of ASO in 151 limbs, TAO in 14, and angiitis (collagen disease) in 26. Although there were no differences in the male-to-female ratio or age between the two groups, the proportion of Rutherford Category- 6 patients was significantly higher in the non-revascularization group $(\mathrm{P}<0.01)$. With respect to concomitant diseases, the incidences of ischemic heart disease $(P=0.11)$, respiratory dysfunction $(P=0.07)$, and malignant tumors $(\mathrm{P}=0.09)$ were higher in the nonrevascularization group, although there were no significant differences between the two groups.

The reasons why revascularization was not performed included heart hypofunction (ejection fraction (EF): $20 \%$ or less, unstable angina, immediately after myocardial infarction, or severe valvular disease) in 50 limbs (26\%), high risks or unfavorable general conditions, such as respiratory dysfunction ( $0.5 \mathrm{~L}$ or less/second), bedridden states, dementia, and cancer-bearing conditions, in $69(36 \%)$, extensive necrosis or infection in 39 (20\%), technical issues, such as the absence of an artery appropriate for anastomosis or an autologous vein for grafting, in 29 (15\%), relief after 
admission in 32 (17\%), and patients' wishes in $12(6 \%)$. In the non-revascularization group, conservative treatment alone was conducted in 122 limbs (64\%), major amputation in $46(24 \%)$ (amputation above the knee: 30 limbs, amputation below the knee: 16 limbs), minor amputation in $14(7 \%)$, and lumbar sympathectomy in $9(5 \%)$.

Of the 306 limbs treated by revascularization, revascularization at a site central to the inguinal region was performed in 44, revascularization up to the proximal popliteal artery in 57, revascularization up to the distal popliteal artery in 23, and revascularization up to the crural or pedal artery in 182 . Of the above 44 limbs, revascularization in the aortic/iliac artery regions was conducted in 38 (bypass alone: 23 limbs, EVT alone: 12, and bypass + EVT: 3 ), and thromboendarterectomy (TEA) or profundaplasty of the femoral artery in 11 . Of 80 limbs treated by bypass of the popliteal artery, revascularization in the aortic/iliac artery regions was performed in 30 (bypass alone: 14 limbs, EVT alone: 13, and bypass + EVT: 3) as simultaneous revascularization on the central side, and TEA/profundaplasty of the femoral artery in 16 . Of 182 limbs treated by crural or paramalleolar bypass revascularization in the aortic/iliac artery regions was conducted in 20 (bypass alone: two limbs, EVT alone: 15, and bypass + EVT: 3) as simultaneous revascularization, TEA of the femoral artery in five, and bypass of the femoral/popliteal arteries in eight.

Of 260 patients (306 limbs) who underwent revascularization, two died within 30 days (early) after surgery (acute liver failure: 1, and non-occlusive intestinal necrosis: 1 ), and one died during admission (liver failure/pulmonary infarction). These patients had received dialysis. The other postoperative complications consisted of heart disease in four patients, arrhythmia in three, pneumonia in two, and cerebral infarction in one. In six patients (six limbs) with early graft failure and four (four limbs) in whom infection control was difficult despite graft patency, major amputation was performed early after surgery (Fig. 1). Of 245 patients (291 limbs) in whom limb salvage could be achieved after revascularization, late graft failure (postoperative patency period: 21 months, range: 2 to 79 months) was confirmed in 28 ( $28 \mathrm{limbs})$. Of these, major amputation was performed in 13 (13 limbs). Of these, five had collagen disease, but this corresponded to $19 \%$ of all patients who underwent surgery for collagen disease-related angiitis. Of patients with graft patency, major amputation was conducted in 13 (13 limbs) due to the progression of ischemia or a difficulty in infection control (postoperative limb salvage period: 7 months, range: 1 to 22 months) (Fig. 2). There were significant differences in the limb salvage rate ( 5 years: 83.5 vs. $55.8 \%$, respectively, $\mathrm{P}<0.0001$ ), survival rate (5 years: 64.4 vs. $56.3 \%$, respectively, $\mathrm{P}=0.001$ ), and AFS ( 5 years: 57.7 vs. $36.0 \%$, respectively, $\mathrm{P}<0.0001$ ) between the revascularization and non-revascularization groups.

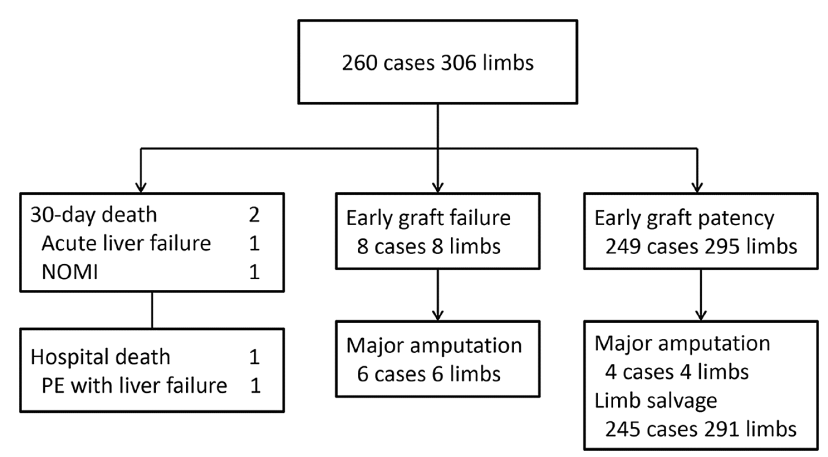

Fig. 1 Early results of critical limb ischemia after revascularization. NOMI: non-occlusive mesenteric ischemia; PE: pulmonary embolism

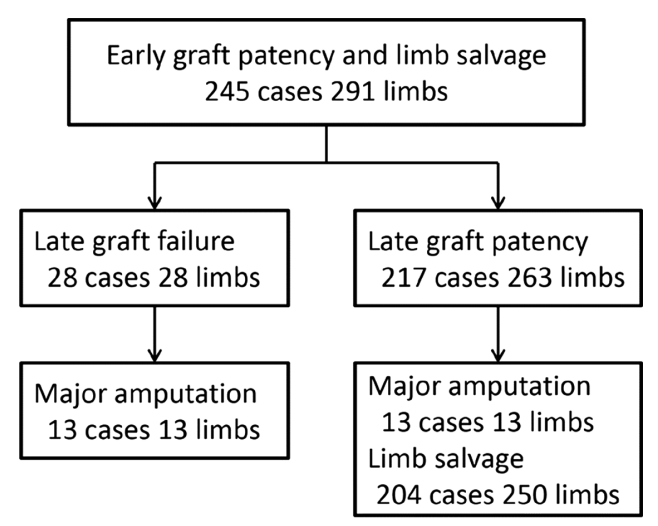

Fig. 2 Long-term results of critical limb ischemia after revascularization.

In the revascularization group, the patency rate with respect to primary diseases was examined only in patients who underwent distal bypass. The 3-year primary patency rates were $80.5 \%$ in patients with ASO and $38.9 \%$ in those with angiitis $(\mathrm{P}<0.0001)$. The 3 -year secondary patency rates were 87.5 and $61.5 \%$, respectively $(\mathrm{P}<0.001)$; the patency rates were significantly lower in those with angiitis. Although there were no significant differences in the survival rate or AFS among all patients with CLI, the 3 -year limb savage rates were $90.0 \%$ in the patients with $\mathrm{ASO}$ and $67.2 \%$ in those with angiitis $(\mathrm{P}=0.041)$ (Fig. 3). The freedom from MALE + POD at three years were 78.0 and $40.8 \%$, respectively ( $\mathrm{P}<0.0001$ ); these percentages were lower in those with angiitis.

Among the patients with ASO, the results were compared between those receiving dialysis (mean age: 64.6 years) and non-dialysis patients (mean age: 71.7 years). The 5-year survival rates were 60.1 and $62.6 \%$, respectively $(\mathrm{P}=0.506)$, showing no significant difference. However, the 5-year limb salvage rates were 73.6 and $94.4 \%$, respectively $(\mathrm{P}<0.0001)$; the percentage was significantly lower in the former (Fig. 4). Furthermore, the 5-year AFS 


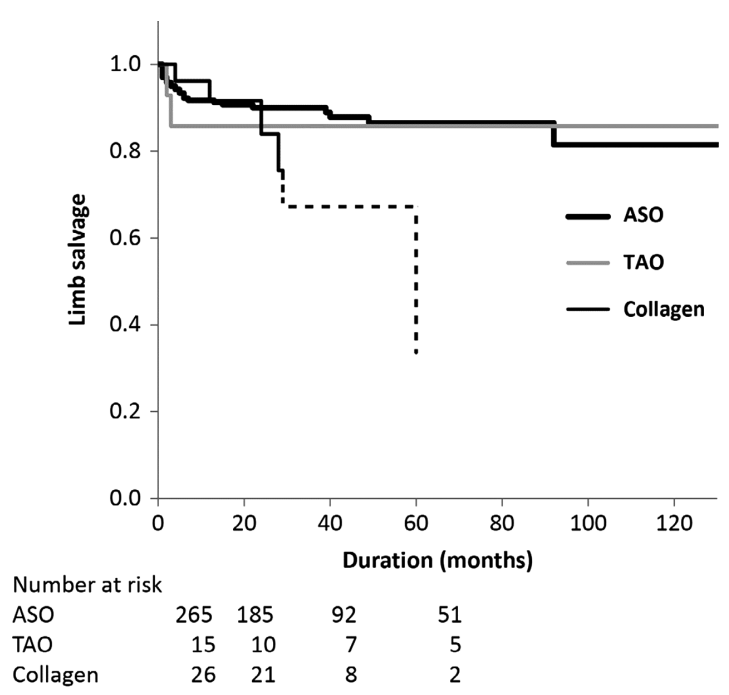

Fig. 3 Cumulative limb salvage rate in critical limb ischemia due to ASO after arterial reconstruction ( $P<0.041$, log-rank test, ASO vs Collagen). Dotted lines represent limb salvage estimates when standard error exceeds 10\%. ASO: arteriosclerosis obliterans; TAO: thromboangiitis obliterans

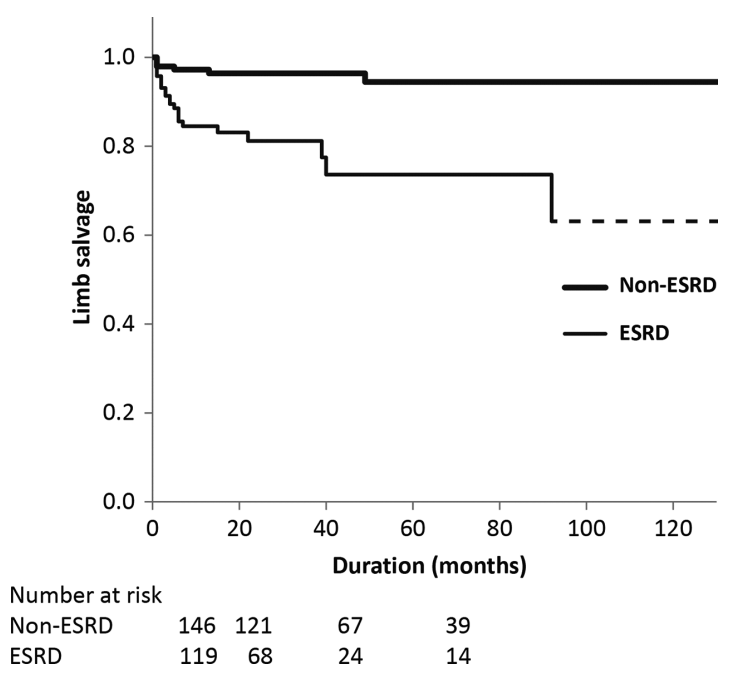

Fig. 4 Cumulative limb salvage rate in critical limb ischemia due to ASO after arterial reconstruction $(\mathrm{P}<0.0001$, log-rank test). Dotted lines represent limb salvage estimates when standard error exceeds 10\%. ESRD: end-stage renal disease; ASO: arteriosclerosis obliterans

was 54.3 and $61.4 \%$, respectively $(\mathrm{P}=0.049)$. The freedom from MALE + POD at five years were 57.1 and $82.9 \%$, respectively $(\mathrm{P}<0.001)$; the prognosis of those receiving dialysis was significantly poorer. On the other hand, the patency rate was compared among ASO patients who underwent distal bypass. The 5 -year primary patency rates in those receiving dialysis and non-dialysis patients were

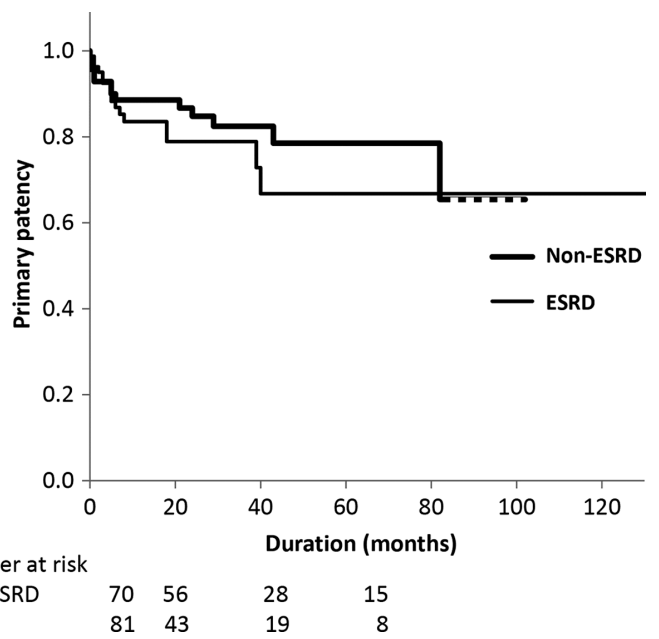

Fig. 5 Cumulative primary patency rate in critical limb ischemia due to ASO after distal bypass ( $P=$ 0.379 , log-rank test). Dotted lines represent primary patency estimates when standard error exceeds 10\%. ESRD: end-stage renal disease; ASO: arteriosclerosis obliterans

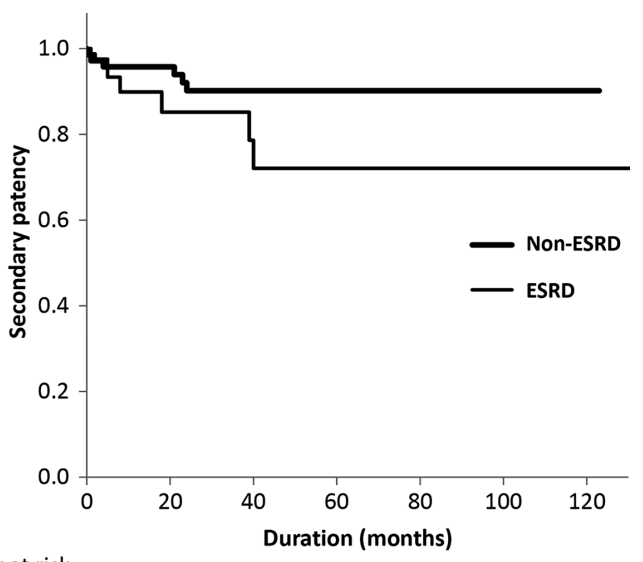

Number at risk

$\begin{array}{llllr}\text { Non-ESRD } & 70 & 61 & 33 & 19\end{array}$

Fig. 6 Cumulative secondary patency rate in critical limb ischemia due to ASO after distal bypass $(P=$ 0.091, log-rank test). ESRD: end-stage renal disease; ASO: arteriosclerosis obliterans

66.8 and $78.5 \%$, respectively $(\mathrm{P}=0.379)$. The 5 -year secondary patency rates were 72.1 and $90.2 \%$, respectively $(\mathrm{P}=0.091)$; the percentages were lower in the former, but there were no significant differences (Figs. 5 and 6).

Proportional hazard analysis was performed using the age, sex, etiology, Rutherford classification, dialysis, smoking, hypertension, diabetes, ischemic heart disease, cerebrovascular disorder, anemia, and serum albumin level as independent risk factors involved in MALE + POD and AFS. Etiologies of angiitis (hazard ratio (HR): 5.10, 95\% 
confidence interval (CI): 2.31-11.26, $\mathrm{P}<0.001)$, dialysis (HR: $2.00,95 \%$ CI: $1.07-3.74, \mathrm{P}=0.031$ ), and ischemic heart disease (HR: 2.00, 95\% CI: 1.10-3.66, P = 0.024) were independent risk factors involved in MALE + POD. Furthermore, independent risk factors involved in AFS consisted of an age of 80 years or older (HR: $2.38,95 \%$ CI: 1.17-4.86, $\mathrm{P}=0.017$ ), an etiology of angiitis (HR: 4.62, 95\% CI: $1.95-10.95, \mathrm{P}<0.001$ ), Category 6 evaluated according to the Rutherford classification (HR: 2.42, 95\% CI: 1.12-5.20, $\mathrm{P}=0.024$ ), dialysis (HR: $2.98,95 \%$ CI: $1.52-5.85, \mathrm{P}=0.002)$, and cerebrovascular disorder (HR: $1.66,95 \%$ CI: 1.14-2.44, $\mathrm{P}=0.009$ ).

\section{Discussion}

CLI is a condition in which not only the limbs but also prognosis is affected. Therapeutic strategies differ from those for claudication. ${ }^{3)}$ To treat CLI, revascularization has been performed if possible. However, actually, it was not conducted in $38 \%$ of CLI patients. In patients with CLI, the proportions of elderly patients, those with a reduction in activities of daily living (ADL), and those with cardiovascular/respiratory disorders or kidney dysfunction are higher than in patients with claudication; revascularization is often impossible from the perspective of the general condition or risks. There are many cases in which either primary amputation or conservative treatment must be selected due to local or technical issues, such as the presence of extensive necrosis or infection and absence of an artery appropriate for bypass or venous graft, regardless of the general condition. When comparing background factors between the revascularization and non-revascularization groups, the proportion of Category- 6 patients evaluated according to the Rutherford classification was significantly higher in the latter, suggesting the importance of treatment in the extended infectionor necrosis-free phase. The most frequent reason why revascularization was not performed was heart hypofunction. In the non-revascularization group, the incidences of ischemic heart disease, respiratory dysfunction, and malignant tumors were higher than in the revascularization group. Recently, strategies to minimize the invasiveness of treatment, such as bypass with nerve block anesthesia, have been devised, but there are still a specific number of patients in whom revascularization is difficult. It is necessary to carefully evaluate whether or not revascularization should be indicated, considering the general condition or prognosis. In the non-revascularization group, the proportion of patients with severe ischemia was significantly higher than in the revascularization group, and both the limb salvage and survival rates were significantly lower. The finding that major amputation reduces survival ${ }^{4)}$ supports this.
Etiological factors for CLI include ASO, Buerger's disease, and angiitis. With respect to primary diseases, the patency rate in patients with collagen disease-related angiitis was lower than in those with ASO, and the limb salvage and the freedom from MALE + POD were also lower. Of our series, the revascularization rates in patients with ASO and collagen disease-related angiitis were 64 and 50\%, respectively. In most patients with angiitis, the targets of bypass, peripheral blood vessels, are absent. Even when revascularization is conducted, long-term results are unfavorable. ${ }^{5)}$ Dermal or subcutaneous arteriolar occlusion may also contribute to such results. For patients with Buerger's disease or angiitis, vascular regenerative treatment is being selected. ${ }^{6-10)}$ In the future, new hybrid therapy may be applied: revascularization for central lesions and vascular regenerative treatment for peripheral lesions.

We compared the results of treatment between dialysis and non-dialysis patients in the revascularization group consisting of patients with ASO. In the former, the limb salvage rate, the freedom from MALE + POD, and AFS were significantly lower than in the latter. However, there was no significant difference in the patency rate. In our study, the 5-year limb salvage and survival rates were 73.6 and $60.1 \%$, respectively, in dialysis patients with ASO. According to previous studies, the incidence of perioperative complications related to revascularization was high in patients with end stage renal disease, ${ }^{11)}$ and the 5 -year limb salvage rate after revascularization was $66.6 \%$. $^{12)}$ The 1 -year limb salvage rate in the absence of gangrene was $74 \%$, whereas that in its presence was $51 \%{ }^{13)}$ Furthermore, the 5 -year survival rate in CLI patients with end stage renal disease is reportedly $23.0 \% ;^{12}$ the prognosis of patients receiving dialysis is poorer than in non-dialysis patients. However, in our study, there was no significant difference in the survival rate between the two groups. In our series, the mean age of patients receiving dialysis was 7 years younger than that of non-dialysis patients, and this may have contributed to the absence of a significant difference. CLI frequently develops in diabetics receiving dialysis. Concomitant diabetes becomes severe with protracted infection, promoting an increase in the area of tissue necrosis; therefore, it is important to perform revascularization before infection involves an extended area. ${ }^{12,13)}$ Of our series, major amputation was conducted due to protracted infection or an increase in the infected area in $6 \%$ of patients with graft patency. It is necessary to carefully achieve limb salvage by sufficient debridement before and after revascularization and postoperative foot care. Calcified lesions in patients receiving dialysis are difficult to manage during bypass/EVT. However, in our study, the patency rate in those receiving dialysis in whom bypass was possible was similar to that in non-dialysis patients; therefore, revascularization should be positively performed, considering postoperative topical infection control. 
Recently, the extent of the lesion area as a criterion to recommend EVT as the first choice in the aortic/iliac artery and femoral/popliteal artery regions was increased in international guidelines for PAD treatment, the Trans Atlantic Inter-Society Consensus II (TASC-II). ${ }^{3)}$ In the BASIL trial, ${ }^{14,15)}$ in which bypass was compared with EVT, there was no difference between the two procedures within 3 years after surgery, but the results for endpoints, such as AFS, in the EVT group reduced thereafter. Based on this, it was concluded that bypass should be recommended for patients with a life expectancy of 2 years or more. For CLI treatment, it is important to review risks/lesions and separately apply or combine the two procedures. If the usefulness of a drug-eluting balloon (DEB) is confirmed through its clinical application in comparison with a drug-eluting stent (DES) in the lower limb field in the future, EVT may be indicated for a larger number of patients to be treated by revascularization for CLI, further increasing the number of treatment options for CLI. In the future, it may become more important to establish a system to promote multidisciplinary treatment, involving foot care, in cooperation with the Department of Vascular Surgery and other departments.

\section{Conclusion}

It is important to treat CLI before the appearance of extensive necrosis or an increase in the infected area. In patients with CLI due to ASO, the results of revascularization are favorable, and revascularization, including bypass, should be positively performed, considering whether or not it should be indicated. In the future, EVT, which is more minimally invasive, may be indicated for a larger number of patients. Furthermore, a multidisciplinary treatment system for CLI should be established in cooperation with several departments or other health care professionals.

\section{Disclosure Statement}

There are no conflicts of interest for the authors.

\section{References}

1) Takayama Y, Takagi A, Sato O, et al. Use of a nondissection method in lower extremity revascularization: a report on our 12-year experience of autogenous vein bypass surgery. Surg Today 1996; 26: 910-4.
2) Rutherford RB, Baker JD, Ernst C, et al. Recommended standards for reports dealing with lower extremity ischemia: revised version. J Vasc Surg 1997; 26: 517-38.

3) Norgren L, Hiatt WR, Dormandy JA, et al. Inter-Society Consensus for the Management of Peripheral Arterial Disease (TASC II). J Vasc Surg 2007; 45 (Suppl S): S5-67.

4) Kalra M, Gloviczki P, Bower TC, et al. Limb salvage after successful pedal bypass grafting is associated with improved long-term survival. J Vasc Surg 2001; 33: 6-16.

5) Deguchi J, Shigematsu K, Ota S, et al. Surgical result of critical limb ischemia due to tibial arterial occlusion in patients with systemic scleroderma. J Vasc Surg 2009; 49: 918-23.

6) Isner JM, Baumgartner I, Rauh G, et al. Treatment of thromboangiitis obliterans (Buerger's disease) by intramuscular gene transfer of vascular endothelial growth factor: preliminary clinical results. J Vasc Surg 1998; 28: 964-73; discussion 973-5.

7) Miyamoto K, Nishigami K, Nagaya N, et al. Unblinded pilot study of autologous transplantation of bone marrow mononuclear cells in patients with thromboangiitis obliterans. Circulation 2006; 114: 2679-84.

8) Moriya J, Minamino T, Tateno K, et al. Long-term outcome of therapeutic neovascularization using peripheral blood mononuclear cells for limb ischemia. Circ Cardiovasc Interv 2009; 2: 245-54.

9) Takahashi M, Ishigatsubo Y, Fujimoto K, et al. Therapeutic angiogenesis by autologous mononuclear cell implantation in patients with intractable vasculitis. J Jpn Col Angiol 2008; 48: 421-4.

10) Kawanaka H, Takagi G, Miyamoto M, et al. Therapeutic angiogenesis by controlled-release fibroblast growth factor in a patient with Churg-Strauss syndrome complicated by an intractable ischemic leg ulcer. Am J Med Sci 2009; 338: 341-2.

11) Kimura H, Miyata $T$, Sato $O$, et al. Infrainguinal arterial reconstruction for limb salvage in patients with end-stage renal disease. Eur J Vasc Endovasc Surg 2003; 25: 29-34.

12) Albers M, Romiti M, De Luccia N, et al. An updated metaanalysis of infrainguinal arterial reconstruction in patients with end-stage renal disease. J Vasc Surg 2007; 45: 536-42.

13) Johnson BL, Glickman MH, Bandyk DF, et al. Failure of foot salvage in patients with end-stage renal disease after surgical revascularization. J Vasc Surg 1995; 22: 280-5; discussion 285-6.

14) Adam DJ, Beard JD, Cleveland T, et al. Bypass versus angioplasty in severe ischaemia of the leg (BASIL): multicentre, randomised controlled trial. Lancet 2005; 366: 1925-34.

15) Bradbury AW, Adam DJ, Bell J, et al. Bypass versus Angioplasty in Severe Ischaemia of the Leg (BASIL) trial: Analysis of amputation free and overall survival by treatment received. J Vasc Surg 2010; 51 (5 Suppl): 18S-31S. 